\title{
A Further Investigation Of Tables Versus Graphs For Decision-Making: Does Accounting Knowledge Make A Difference?
}

Joel M. Strong, Ph.D., CPA, (E-mail: jmstrong@stcloudstate.edu), St. Cloud State University Kris Portz, Ph.D., CPA, (E-mail: ksportz@stcloudstate.edu), St. Cloud State University

\begin{abstract}
This research uses cognitive processing and cognitive induction theory to examine whether accounting knowledge affects a decision-maker's performance when using tables or graphs to perform a bankruptcy prediction task. A laboratory experiment was conducted using a $2 x 2$ betweensubjects design where accounting knowledge (low vs. high) is crossed with information presentation format (tabular vs. graphical). The results suggest that accounting knowledge does affect the use of alternative information presentation formats.
\end{abstract}

\section{Introduction}

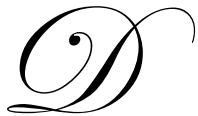

evelopments in information systems technology make displaying accounting information graphically a practical alternative to traditional tabular presentations (Davis 1989). Given that technology allows us to easily change and manipulate the way in which information is presented, the critical element now becomes a question of which format improves decision-making and how do presentation choices affect user judgment. For instance, do graphics or pictorials increase communication effectiveness over more traditional formats when presenting financial accounting information?

While a significant amount of research has explored the decision usefulness of graphs for increased communication effectiveness, nearly two decades of investigation has found little empirical evidence supporting the hypothesized benefits of graphs. The findings of the information presentation research, in both the management information systems (MIS) and accounting literature, are inconclusive and often in conflict with each other. Therefore, the purpose of this study is to examine a possible explanation for some of the conflicting results in the prior studies. This study uses cognitive processing theory of induction (Holland et al. 1986) and cognitive fit theory (Vessey 1991) to introduce and support a new variable, accounting knowledge. Specifically, we hypothesize that an individual's level of accounting knowledge affects decision-making performance when using different presentation formats to perform a bankruptcy prediction task.

Cognitive fit theory suggests that a decision-maker's performance depends both on how the information is presented to the decision-maker and the task at hand. Cognitive fit theory also suggests that certain tasks are better suited for certain presentation formats. When type of information presentation and type of task are appropriately matched, decision-making will be enhanced. Prior research has aimed at validating cognitive fit theory by investigating the effectiveness of tables versus graphs for various decision-making tasks. Consistent with cognitive fit theory, authors hypothesize that tables are more effective when performing symbolic tasks and graphs are more effective when performing spatial tasks. Unfortunately the results have often been inconclusive and conflicting.

Readers with comments or questions are encouraged to contact the authors via email. 
Most researchers accept that despite the conflicting results, task does moderate the relationship between information presentation format and decision-making effectiveness (DeSanctis 1984; Davis 1989; Amer 1991; Vessey 1991. However, to help sort out the conflicting results of prior studies and to make further progress in this area, researchers have suggested a need to look at other variables, in addition to information presentation and task, which may influence decision-making effectiveness (i.e. Frownfelter 1998). For instance, Goodhue and Thompson (1995) suggest that individual characteristics such as training, experience, and motivation may also influence cognitive fit. This study considers the suggestions of previous authors by introducing a new variable into the cognitive fit framework. Namely, this study investigates if accounting knowledge, an individual characteristic, affects the decisionmaker's ability to use different presentation formats during a decision-making task. We predict that accounting knowledge will increase an individual's ability to use tabular information presentations when performing spatial tasks. Recall that cognitive fit theory predicts that graphs are more effective for spatial tasks.

To investigate this issue, participants ( 87 accounting students) performed a bankruptcy prediction task (spatial task). Each subject was required to predict the bankruptcy of 20 firms (10 that went bankrupt and 10 that did not go bankrupt). Our results suggest that accounting knowledge does influence an individual's ability to use tabular information presentations for spatial tasks.

\section{Research Model and Hypotheses Development}

The research model used in this study is based on a combination of cognitive fit theory and cognitive induction theory. In this section of the paper we will describe each of these theories, build a research model, and use the discussion to develop hypotheses to be empirically tested.

\subsection{Cognitive Fit Theory}

Cognitive fit theory suggests that when the types of information emphasized in the decision (problem representation) and task match, the problem-solver can use processes to formulate a mental representation that also emphasizes the same type of information (Vessey 1991). Consequently, the process the problem-solver uses to both act on the decision and the task will match. This match, which is consistent with the mental representation, facilitates the decision-making process. Figure 1 illustrates the notion of cognitive fit. This model views problem solving as the outcome of the relationship between the problem representation (i.e. tabular vs. graphical), the decisionmaking task, and the individual's mental representations. Cognitive processes are represented by the arrows linking pairs of elements in the model. The mental representation is the way the problem-solver represents a problem in human working memory.

Figure 1 Model of cognitive Fit

(Adapted from Vessey (1991))

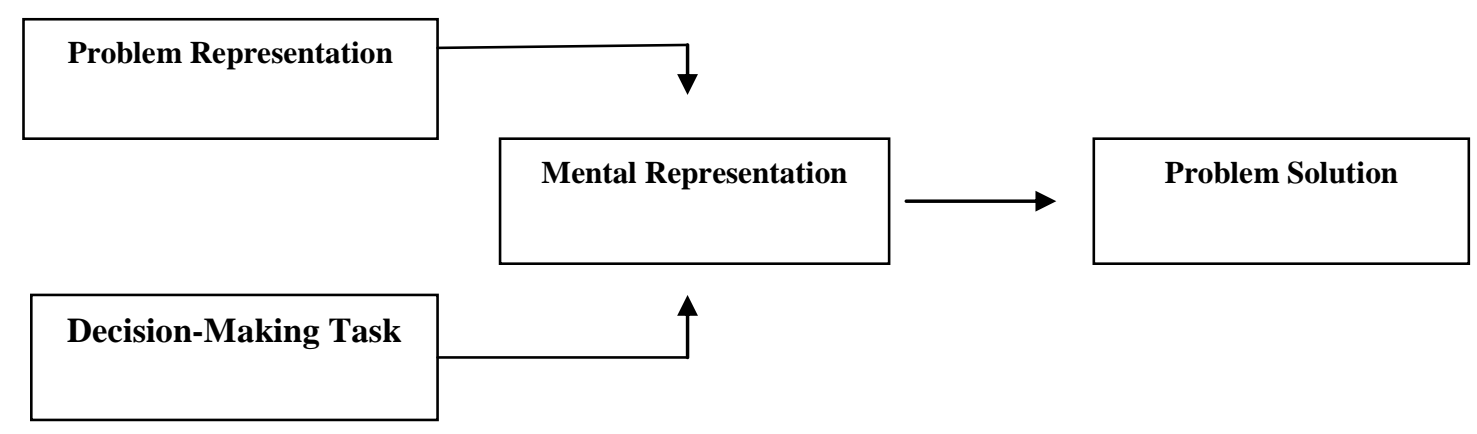

Vessey (1991) identifies two basic types of cognitive processes that are needed to solve problems: spatial and symbolic. Tasks that emphasize spatial processes (spatial tasks) are those that require the decision-maker to as- 
sess the problem area as a whole rather than as discrete data values. These types of tasks require making associations or perceiving relationships in the data. Some examples of spatial tasks would be to (1) compare two data values, (2) compare trends, or (3) assess relationships in the data. Alternatively, tasks that emphasize symbolic processes (symbolic tasks) involve the extraction and evaluation of discrete, therefore precise, data values, often requiring computation and analysis.

Vessey (1991) suggests that graphs are more congruent with tasks that emphasize spatial processes because graphs are spatial representations that emphasize relationships in the data rather than discrete values. Tables are more congruent with tasks that emphasize symbolic processes because tables present discrete data values that can be used directly in analytical and computational processes. Performance will be enhanced when there is a cognitive fit or match between the presentation of information and task type. As long as there is a complete fit of representation, processes, and task type, each representation will lead to both quicker and more accurate problem solving. When a mismatch occurs between the task and the information presentation, additional cognitive acts are required to reach a conclusion.

Cognitive fit results in more effective and efficient problem solving. However, a spatial representation does not have to be used to solve a spatial task, nor does a symbolic representation have to be used to solve a symbolic task. When the information in the problem representation and the task do not match, similar processes cannot be used to both act on the problem representation and solve the problem, and the mental representation will have to be transformed. The overall effect will be an increase in the variability of the accuracy, resulting in less effective problem solving (Vessey 1994).

\subsection{Cognitive Induction Theory}

Cognitive theory of induction ${ }^{1}$ (Hayes-Roth et al. 1983; Anzai and Yokoyama 1984; Glaser 1984; Holland et al. 1986; Vera-Munoz 1998) provides a possible basis for understanding the role of accounting knowledge and its possible effect. This theory suggests that existing knowledge structures stored in a decision-maker's memory influence how external cues are combined with information available in memory to form a mental representation of the problem. Figure 2 illustrates how accounting knowledge fits into the notion of cognitive theory of induction. Accounting knowledge structures are likely to include both the double-entry representation for historical transactions, skeletal forms of the traditional financial statements, traditional ratio analyses techniques, (Waller and Felix 1984), and analytical judgement skills that are developed through problem-solving tasks in the formal accounting education process. In contrast, accounting knowledge typically does not include information in the form of graphical presentations. The quality, completeness, and coherence of the problem-solvers' initial mental model determine the effectiveness of decision-making.

Figure 2 Cognitive Theory of Induction

(Adapted from Holland et. al. (1986))

\begin{tabular}{|c|c|c|}
\hline $\begin{array}{l}\text { Accounting Knowledge } \\
\text {-High } \\
\text {-Low }\end{array}$ & $\begin{array}{l}\text { Mental Mod- } \\
\text { el/Representation of the Ac- } \\
\text { counting Decision-Making } \\
\text { Task }\end{array}$ & $\begin{array}{l}\text { Decision-Making } \\
\text { Performance } \\
\text {-Effectiveness }\end{array}$ \\
\hline
\end{tabular}




\subsection{Research Model}

As was described in the preceding two sections, cognitive fit theory views problem-solving as the outcome of the relationship between the problem representation, the problem-solving task, and the individual's mental representation. Application of the cognitive theory of induction suggests that, when presented with information to assist in an accounting decision-making task, decision-makers with high accounting knowledge will construct a mental model, which is a mental representation of their accounting knowledge. Together, these two theories form the framework that will be used as the basis for the hypotheses to be tested in this study. Figure 3 depicts this framework.

Figure 3 Research Model of the Inductive Framework within the Theory of Cognitive Fit.

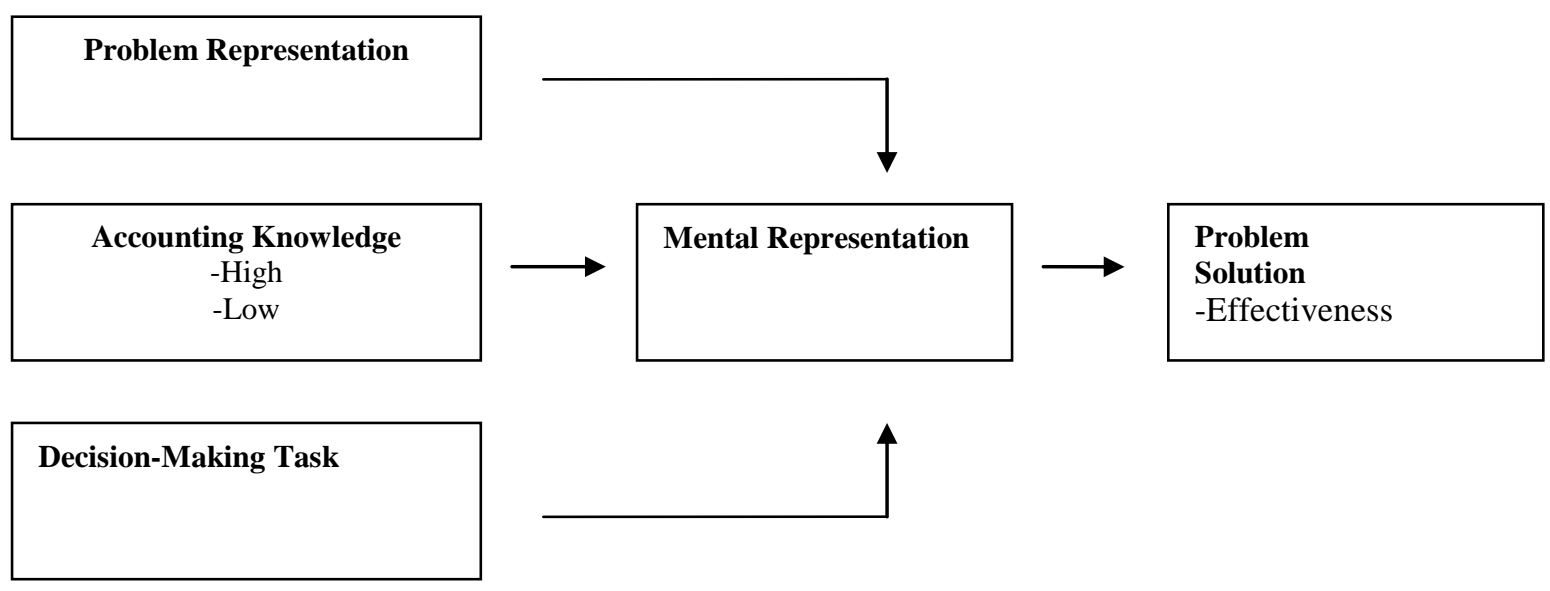

\subsection{Hypotheses}

The bankruptcy prediction task used in the current study is considered a spatial task, where comparing data values, assessing trends and considering relationships in the data is required. Therefore, the decision-maker should perform the bankruptcy prediction task better with graphs than tables. If the decision-maker uses tables, a mismatch occurs between the information presentation and the information-processing requirements of the task. Therefore, task complexity increases, resulting in a more difficult task and decreased performance. However, in this study high-accounting knowledge is hypothesized to allow the individual to overcome the increased task complexity and perform at the same level as if using graphs.

High-accounting knowledge decision-makers should be able to use tables just as effectively as graphs and overcome any predicted benefits of graphs for this task because of their stored accounting knowledge (which is more conducive to tables). In order to examine the effects of high accounting knowledge on an individual's performance using tables versus graphs, the following hypothesis is proposed:

Hypothesis 1: There will be no difference in decision effectiveness for high accounting knowledge subjects using graphical information presentations, as opposed to tabular information presentations, when performing a multiattribute accounting decision-making task.

In the absence of high accounting knowledge, decision-makers should be able to generate a mental representation of the task that is consistent with the problem representation of the task. With the bankruptcy prediction task used in this study, graphs should provide decision makers with a better cognitive fit. Therefore, without the 
impact of prior accounting knowledge structures, low accounting knowledge subjects will make more effective judgments using a graphical information presentation. In order to examine the effects of low accounting knowledge on an individual's performance, the following hypothesis is proposed:

Hypothesis 2: Subjects with low accounting knowledge will make more effective decisions using graphical information presentations, as opposed to a tabular information presentation, when performing a multiattribute accounting decision-making task.

\section{Research Methodology}

The research hypotheses are addressed with a between-subjects experiment in which subjects are presented with a bankruptcy prediction task (a spatial task) where the financial information presented either in the form of tables, or equivalent financial information in the form of line graphs or bar graphs. The bankruptcy prediction task used in the current study was adapted from Umanath and Vessey (1994). Each subject was required to predict the bankruptcy of twenty firms (10 that went bankrupt and 10 that did not go bankrupt). Each subject was provided with either a graphical or tabular display of five years of financial data and was asked to predict bankruptcy in the sixth year. The financial data used in the current study are debt service, total assets, current ratio, total owner's equity, accounts receivable turnover, and inventory turnover. ${ }^{2}$

The hypotheses are tested using ANCOVA. The experimental design provides randomization of information presentation treatments, and attempts to control for the potential effect of cognitive style/ability and finance knowledge on performance. The dependent variable of interest is the accuracy of the bankruptcy predictions (effectiveness).

The effects of accounting knowledge are addressed by selecting subjects from two groups likely to possess distinctly different levels of accounting knowledge, based on the number of college accounting course hours completed. The low-accounting knowledge level is composed of undergraduate students, who have no significant accounting experience, and have not completed more than six hours of accounting courses. The high-accounting knowledge level is composed of undergraduate students, who also have no significant accounting experience, but have completed at least twelve hours of accounting courses. A total of 87 subjects participated in the study. Experiment participants were recruited from the undergraduate accounting program at a large Midwestern University. The sample of 87 subjects was composed of 42 low-accounting knowledge subjects and 45 high-accounting knowledge subjects.

The information presentation factor was manipulated by requiring subjects to make bankruptcy predictions using financial information in one of two different presentation formats (tables or graphs). The first level of presentation format, categorized as tabular, is financial ratios, presented in the form of numbers or percentages. The second level of presentation format, categorized as graphical, is line graphs and bar graphs. Conventional financial market practice is used such that financial statement balances are represented as bar graphs and financial ratios are represented as line graphs. These formats are consistent with the types of graphs used in prior research (Vessey and Galletta 1991; Umanath and Vessey 1994). The graphs were constructed to provide quality graphics, thereby avoiding the introduction of graph quality as a confounding variable.

Prior studies on presentation format suggest a positive association between performance in multiattribute accounting tasks and cognitive style/ability (Andersen and Reckers 1992; Benbasat and Dexter 1985; Nibbelin et al. 1992; Frownfelter 1998). In the present study, cognitive style/ability is likely to affect subjects' bankruptcy predictions. Thus, the potential effect of cognitive style/ability on decision-making performance is controlled in this study by estimating and statistically removing the effect of cognitive style/ability. We use the Embedded Figures Test to measure cognitive style/ability (Witkin et al. 1971). This study makes no a priori predictions with respect to differences in cognitive style/abilities between high and low-accounting knowledge individuals.

We also control for the number of college finance hours. This variable is not of interest in the current study but must be controlled to avoid confounding problems. Frownfelter (1998) found significant results for the number 
of finance credits completed by the subjects in her study. Finance knowledge is similar to accounting knowledge in that its primary focus is on analytical problem-solving skills using information in the form of traditional tabular financial statements and tabular ratio presentations. Therefore, this variable will be measured directly, by asking subjects the number of finance credit hours they have completed as part of the pre-experimental questionnaire, and included in the statistical model as a control variable.

Finally, the dependent variable of interest is the effectiveness of bankruptcy predictions. Effectiveness was measured as decision accuracy, the number of correct bankruptcy predictions.

\section{Results}

\subsection{Manipulation Checks}

Two assumptions were made regarding the experiment. First, any performance differences must be attributed to the level of accounting knowledge and not to other confounding differences between the two groups of experimental subjects. This study hypothesizes that accounting knowledge increases an individual's ability to use tabular information when performing a spatial task. The experimental differences expected in this study should be due to the level of accounting knowledge and not differences in task specific knowledge. Therefore, the subject's task specific knowledge was expected to be equal, on average, across subject groups (high-accounting knowledge vs. low-accounting knowledge). As part of the post experimental questionnaire, subjects were given eight multiplechoice questions to measure task specific financial ratio analysis knowledge. The mean score for the lowaccounting knowledge group was 4.69 while the score for the high-accounting knowledge group was 4.80 . As expected, there was no significant difference between the subjects in the two knowledge groups.

Second, cognitive fit theory says that there is a cognitive fit between spatial tasks and graphical information presentations. Therefore, it is extremely critical to the current study that the bankruptcy prediction task is perceived as a spatial task. In order to measure this criterion, subjects answered six questions related to task perceptions. Three questions measured spatial task perceptions and three questions measured symbolic task perceptions. These variables were measured on a seven point Likert scale anchored at one end by agree (Likert scale of 1) and at the other end by disagree (Likert scale of 7). The subjects, on average, tended to agree more with the questions pertaining to the spatial task questions than symbolic task questions. The various paired comparisons between the spatial and symbolic task questions are all highly significant $(\mathrm{p}=.000)$. These data suggest that subjects tended to view the task as a spatial task where performance is expected to be better for those subjects using graphs.

\subsection{Test of the Effectiveness Hypotheses}

Two hypotheses are formed regarding bankruptcy prediction accuracy. A graphical representation of the bankruptcy prediction accuracy is presented in Figure 4

Descriptive results and the ANCOVA are presented in Table 1, panel A and B, respectively. The results indicate a main effect for information presentation and a covariate effect for finance hours.

Panel C of Table 1 reports the results of the planned comparisons for purposes of testing H1a and H2a, after controlling for cognitive style/ability and finance hours. ${ }^{3}$ H1a predicts that there will be no difference in the accuracy of bankruptcy predictions for high-accounting knowledge subjects using graphical information presentations, as opposed to using tabular information presentations (cell 1 vs. cell 2, Table 1, panel A). The planned comparison for testing H1a shows that the effect of information presentation in the high-accounting knowledge level, after controlling for cognitive style/ability and finance hours, is not significant $(t=.16, p=.57)$. This result indicates that, on average, bankruptcy prediction accuracy did not differ between presentation styles for subjects with high-accounting knowledge. 


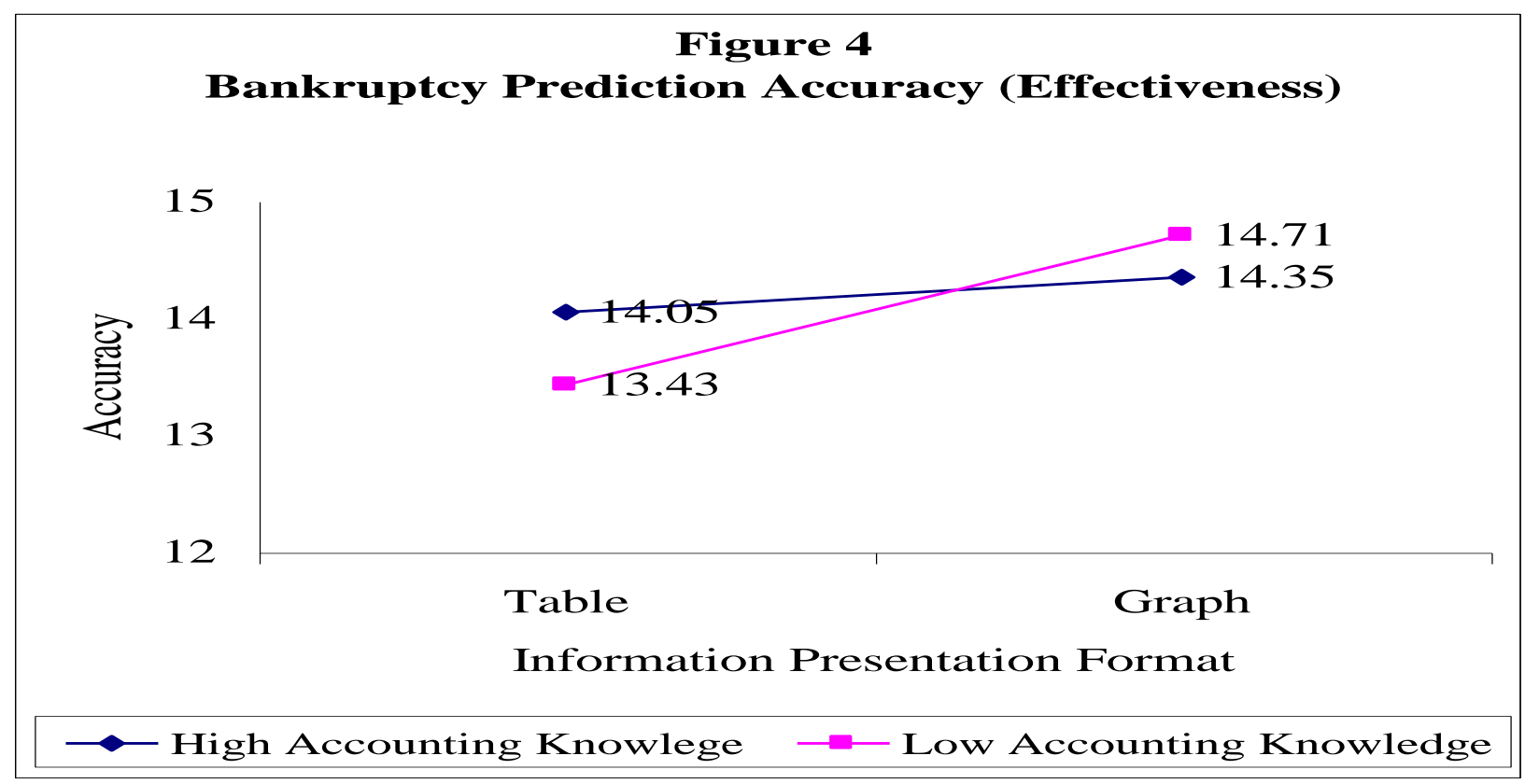

Hypothesis 2a predicts that low-accounting knowledge subjects will make more accurate bankruptcy predictions using graphical information presentations, as opposed to using tabular information presentations (cell 3 vs. cell 4, Table 1, panel A). The planned comparison for testing $\mathrm{H} 2 \mathrm{a}$ is presented in Table 1, panel C. As predicted, the effect of information presentation in the low-accounting knowledge level, after controlling for cognitive style/ability and finance hours, is significant $(\mathrm{t}=2.32, \mathrm{p}=.05)$. This result indicates that, on average, bankruptcy prediction accuracy was greater for subjects in the low-accounting knowledge level using graphical information presentations than for subjects using tabular information presentations. With a low-level of accounting knowledge, the decision-maker forms a better cognitive fit between the task and the graphical presentation consistent with the theory of cognitive fit.

The results indicate that high-accounting knowledge individuals perform equally well on a spatial task using tables or graphs. They appear to use their accounting knowledge structures to overcome the mismatch created by the use of tables for a spatial task and perform equally well using graphs. In contrast, low-accounting knowledge individuals do realize the benefits of graphical information presentation and perform significantly better using graphs when making bankruptcy predictions. Taken together, it appears that accounting knowledge creates or refines the existing knowledge structures stored in an individual's long-term memory, which influence how available information is combined to form a mental model of the problem.

\section{Discussion and Conclusions}

This paper examines the effect of accounting knowledge on the use of tabular and graphical information presentations in an accounting decision-making task. One of the most important reasons cited for the unequivocal results in the presentation format literature is that most studies do not offer a sound a priori basis, theoretical or otherwise, as to why a given information presentation would lead to better performance in a given task setting. Recent work by Vessey (1991) has attempted to apply cognitive fit theory to this research. However, results of subsequent research attempting to test this theory have been mixed (Amer 1991; Nibbelin et al. 1992; Frownfelter 1993; Umanath and Vessey 1994;Tuttle and Kershaw 1998). The results of these studies suggest the continuing need for theory development, particularly identifying other variables that may be relevant to this type of research (Frownfelter 1998). 
Accounting knowledge appears to be an important variable in presentation format research, certainly one worthy of investigation. This research uses a bankruptcy prediction task similar to prior research. However, the distinguishing characteristic of this study is an experimental design that includes knowledge differences between accounting decision-makers and non-accounting decision-makers. The results of the study are important for exploring accounting knowledge and improving our understanding of the theory underlying information presentation effects.

The study extends the existing research by providing a more complete understanding of the effects of cognitive fit and information presentation on performance in an accounting decision-making task. The effect of accounting knowledge allows inferences about knowledge differences to be made. Consistent with theory, lowaccounting knowledge subjects made more accurate bankruptcy predictions using graphs than tables. In contrast, there were no significant differences between graphs and tables for high-accounting knowledge subjects.

The results indicate that high-accounting knowledge individuals perform equally well on a spatial task using graphs as they do using tables. In contrast, low-accounting knowledge individuals do realize the benefits of graphical information presentation and perform significantly better using graphs. Taken together, accounting knowledge seems to create or refine the existing knowledge structures stored in an individual's long-term memory, which influence how available information is combined to form a mental model of the problem.

The main contribution of the study is a more complete understanding of the effect of accounting knowledge and information presentation format on decision-making performance. These results should help to improve our understanding of cognitive fit theory as it pertains to the information presentation literature. This study provides initial evidence that individual characteristics influence cognitive fit. Future research, which relies on the theory of cognitive fit, should consider the influence of other individual characteristics.

The findings of this study may also help to explain, in part, the inclusive results reported in prior research when high-accounting knowledge subjects were used. The results of this study lead us to believe that the results reported in the prior literature may have been driven by the use of high-accounting knowledge individuals.

Finally, the effects of variations in display mode is a particularly timely topic. We are currently in the infancy of an information revolution that will change the way we view information. This revolution will free information from paper and put the information on the screens of computer users. A substantial number of corporations are publishing a wealth of financial information about themselves, including annual and quarterly reports and fillings with the Securities and Exchange Commission, on the World Wide Web. The Internet gives companies far more options than print, including plenty of space for graphic presentations of its financial information. As we progress through this information revolution, the effectiveness of different modes of information presentation will become a relevant issue.

\section{Limitations}

The impact of accounting knowledge and information presentation format on decision-making is explored using a laboratory experiment with student subjects. Some of the limitations in this study are inherent in the methodology, others are inherent in the choices and compromises of the researcher. Any conclusion drawn from this study must be relied on only with full knowledge of the limitations.

External validity may be limited because of the experimental methodology used. Thus, the results of the study may not be generalizable beyond the (1) choice of subjects, (2) task used in the experiment, (3) formats used, and (4) the specific financial information used. Additional limitations concern the choices of the levels tested for the independent variables and the measurement instruments used to measure both the extraneous variables and the dependent variables. Finally, this proposal examines only subjects' performance in determining the effect of accounting knowledge and information representation. An alternative method that can be used in determining knowledge structure would be to observe the process used by subjects in performing the bankruptcy prediction task. 


\section{Suggestions for Future Research}

Several possibilities for future research exist. First, while this study measures performance, an alternative is to observe the processes used by subjects with varying levels of accounting knowledge when the information presentation is manipulated. This could be accomplished using a process tracing or a verbal protocol technique. Computer software could be used to collect information regarding the decision processes used by decision-maker using different information presentations. This will give a more complete representation of how decision-makers make decisions in addition to how well they make them.

Second, since the effects of the information presentation may vary with environmental factors, decisionmaker characteristics, and task structure, a realistic systems development alternative is to develop flexible systems that allow the decision-maker to choose the information presentation mode that they would like to use in a problemsolving task. A flexible system design has the potential to facilitate individual judgment strategy adoption and improve the cognitive fit between the task, the individual, and the information presentation. In order to accomplish this goal, further research is necessary to determine if the decision-maker will choose the appropriate presentation mode to match their problem-solving strategy in a particular situation. The results of such studies could be used to develop information systems that are flexible to the individual in different task settings under various environmental conditions.

Finally, researchers may want to investigate the use of graphics for other purposes besides decision aids. For example, it would be interesting to see the impact of presentation format on the persuasion of an individual or group of individuals. This type of research would be highly relevant to public accounting. Accounting firms spend a great deal of time and money making presentations in an effort to sell their services. Maybe a graphical presentations or dynamic graphical presentation serves as a better persuasion tool than the typical proposal format currently being used by most firms. This is all made possible by such software packages as Microsoft PowerPoint, Corel Presentation, and Lotus Freelance.

${ }^{1}$ The cognitive processing theory used in this study is one way to view the world. The study does not test the cognitive theory of induction per se, but uses its framework to guide the research.

${ }^{2}$ These ratios were selected from prior information presentation research (Umanath and Vessey 1994) and from the financial accounting literature dealing with bankruptcy predictions (i.e., Casey 1980; Ohlson 1980; Johnson 1983). The number of financial ratios was limited to six in order to avoid the confounding effects of information overload on the results of the current study.

${ }^{3}$ One assumption that the ANCOVA model makes is that the covariate effect is equal across all treatment (i.e., equal slopes). The homogeneity of slope assumption for the cognitive style/ability and finance hour's covariates was tested using the Levene test (SPSS 1999, 124-125). The results do not allow rejection of the null hypothesis that the slopes for the covariates, cognitive style/ability $(\mathrm{F}=1.97, \mathrm{p}=.16)$ and finance hours $(\mathrm{F}=.05, \mathrm{p}=.83)$, are homogeneous. Since the slopes are assumed to be equal across all treatments, the study proceeds with a standard ANCOVA analysis.

\section{References}

1. Amer, $\mathrm{T}$, "An experimental investigation of multi-cue financial information display and decision making," The Journal of Information Systems, Vol. 5, pp.18-34, 1991.

2. Anderson, J. C. and P. M. J. Reckers, "An empirical investigation of the effects of presentation format and personality on auditor's judgment in applying analytical procedures," Advances in Accounting, Vol. 10, pp.19-43, 1992.

3. Anzai, Y., and T. Yokoyama, "Internal models in physics problem solving," Cognition and Instruction, Vol. 1, 397-450, 1984.

4. Benbasat, I. and A.S. Dexter, "An experimental evaluation of graphical and color-enhanced information presentation," Management Science, Vol. 31, pp.1348-1364, 1985. 
5. Casey, C. J., "The usefulness of accounting ratios for subjects' predictions of corporate failure: Replication and extensions," Journal of Accounting Research, Vol. 18, pp.603-613, 1980.

6. Davis, L. R., "Report format and the decision maker's task: an experimental investigation," Accounting Organizations and Society, Vol. 14, pp.495-508, 1989.

7. DeSanctis, G., "Computer graphics as decision aids: Directions for research," Decision Sciences, Vol.15, pp. 463-87, 1984.

8. Frownfelter, C. A., "The effects of differing information presentation of general purpose financial statements on users' decisions," Journal of Information Systems, Vol. 12, No.2, pp. 99-107, 1998.

9. Glaser, R., "Education and thinking: The role of knowledge," American Psychologist, Vol. 39, pp. 93-104, 1984.

10. Goodhue, D. L. and R. L. Thompson, "Task-technology fit and individual performance," MIS Quarterly, Vol. 19, pp. 213-237, 1995.

11. Hayes-Roth, F., D. Waterman, and D. Lenant, Building Expert Systems, Addison-Wesley, Reading, MA, 1983.

12. Holland, J., K. Holyoak, R. Nisbett, and P. Thagard, Induction: Processes of Inference, Learning, and Discovery, MIT Press, Cambridge, MA, 1986.

13. Johnson, B. W., "Representativeness in judgmental predictions of corporate bankruptcy," The Accounting Review, Vol. 58, pp.78-97, 1983.

14. Nibbelin, M. C., C. D. Bailey, and R. W. Zmud, "The effects of mode of information presentation and cognitive style on bond-rating change decisions," Advances in Accounting, Vol. 10, pp. 159-174, 1992.

15. Ohlson, J. A., "Financial ratios and the probabilistic prediction of bankruptcy," Journal of Accounting Research, Vol. 18, pp.109-131, 1980.

16. SPSS. SPSS for Windows—Base System Applications Guide (Release 9.0). Chicago, IL: SPSS Inc., 1999.

17. Tuttle, B. M. and R. Kershaw, "Information presentation and judgment strategy form a cognitive fit perspective, Journal of Information Systems, Vol. 12, pp.1-17, 1998.

18. Umanath, N. S. and I. Vessey, "Multiattribute data presentation and human judgment: A cognitive fit perspective," Decision Sciences, Vol. 25, pp.795-824, 1994.

19. Vera-Munoz, S. C., "The effects of accounting knowledge and context on the omission of opportunity costs in resource allocation decisions," The Accounting Review, Vol. 73, pp. 47-72, 1998.

20. Vessey, I, "Cognitive Fit: A theory-based Analysis of the graphs versus tables literature," Decision Sciences, Vol. 22, pp.219-237, 1991.

21. _The effect of information presentation on decision making: A cost-benefit analysis," Information \& Management, Vol. 27, pp.103-119, 1994.

22. Vessey, I. and D. Galletta, "Cognitive fit: An empirical study of information acquisition," Information Systems Research, Vol. 2, pp.63-84, 1991.

23. Waller, W. and S. Felix, "The auditor and learning from experience: Some conjectures," Accounting, Organizations and Society, Vol. 9, pp. 383-408, 1984.

24. Witkin, H. A., D. R. Goodenough, and P. K. Oltman, "Psychological differentiation: Current status," Journal of Personality and Social Psychology, Vol. 37, pp.1127-1145, 1979. 